\title{
Alterations of regional homogeneity in pediatric bipolar depression: a resting-state fMRI study
}

\author{
Weijia Gao ${ }^{1,2 \dagger}$, Qing Jiao ${ }^{3 \dagger}$, Shaojia Lu $^{4 \dagger}$, Yuan Zhong ${ }^{5,6}$, Rongfeng Qi ${ }^{5}$ Dali Lu', Qian Xiao ${ }^{1}$, Fan Yang ${ }^{1}$, \\ Guangming Lu ${ }^{5^{*}}$ and Linyan Su ${ }^{1 *}$
}

\begin{abstract}
Background: Pediatric bipolar disorder (PBD) has attracted increasing attentions due to its high prevalence and great influence on social functions of children and adolescents. However, the pathophysiology underlying PBD remains unclear. In the present study, the resting-state functional magnetic resonance imaging (fMRI) was used to detect abnormalities of baseline brain functions in depressed PBD youth.

Methods: Seventeen youth with PBD-depression aged 10 - 18 years old and 18 age- and sex-matched normal controls were recruited in this study. The fMRI data under resting state were obtained on a Siemens 3.0 Tesla scanner and were analyzed using the regional homogeneity (ReHo) method. Correlations between the ReHo values of each survived area and the severity of depression symptoms in patients were further analyzed.

Results: As compared with the control group, PBD-depression patients showed decreased ReHo in the medial frontal gyrus, bilateral middle frontal gyrus and middle temporal gyrus, and the right putamen. Significant negative correlations of the mood and feelings questionnaire scores with mean ReHo values in the medial frontal gyrus and the right middle frontal gyrus in PBD-depression patients were observed.

Conclusion: Our results suggest that extensive regions with altered baseline brain activities are existed in PBD-depression and these brain regions mainly locate in the fronto-limbic circuit and associated striatal structures. Moreover, the present findings also add to our understanding that there could be unique neuropathophysiological mechanisms underlying PBD-depression.
\end{abstract}

Keywords: Pediatric bipolar disorder (PBD), Depression episode, Resting-fMRI, Regional homogeneity (ReHo)

\section{Background}

Bipolar disorder $(\mathrm{BD})$ is one of the major psychiatric illnesses which can be appeared across all age groups including children and adults. BD which may lead to emotional and cognitive function impairments [1] is always characterized by alternating mood state between mania or hypomania, and depression. As compared with adult $\mathrm{BD}$, pediatric $\mathrm{BD}(\mathrm{PBD})$ often shows more atypical symptoms and comorbidities [2]. Although mania is the

\footnotetext{
*Correspondence: cjr.luguangming@vip.163.com; su-linyan@hotmail.com ${ }^{\dagger}$ Equal contributors

${ }^{5}$ Department of Medical Imaging, Jinling Hospital, Clinical School of Medical College, Nanjing University, 305 Zhongshan East Road, Nanjing 210002, Jiangsu, China

${ }^{1}$ Mental Health Institute of The Second Xiangya Hospital, National

Technology Institute of Psychiatry, Key Laboratory of Psychiatry and Mental Health of Hunan Province, Central South University, No. 139 Renmin Road, Changsha 410011, Hunan, China

Full list of author information is available at the end of the article
}

hallmark characteristic of $\mathrm{BD}$, much more evidence suggests depression episode make a major contribution to the disability of BD patients [3]. It has been reported that BD patients spend more time in depression [4] and depression may cause greater influence on quality of life, risk of suicide, and life-long progression [5] in BD patients. To date, plenty of studies have been directed towards etiology, neurobiology, and pathogenesis of $\mathrm{BD}$, however, the mechanisms responsible underlying the development of $\mathrm{BD}$ remain unclear.

In the last two decades, there has been a considerable amount of fMRI studies for adult BD pointing to a relevant role of brain functional abnormalities in the pathophysiology of the disease. Most of the studies have focused on altered brain activations in the cortico-limbic pathways which are responsible for emotional regulation [6,7]. The most consistent fMRI findings in previous studies for BD 
are hypoactivation of the frontal lobe [8], hyperactivation of the limbic structures $[9,10]$ and abnormal connectivity between frontal and limbic structures [11,12]. In this context, the dysregulation of mood and developments of extreme mood states in BD patients are always attributed to disruptions of prefrontal modulations of limbic structures [7].

Although many studies have focused on baseline brain functional changes in $\mathrm{BD}$ patients, most studies have investigated adult $\mathrm{BD}$, and relatively less research on PBD has been published. To our knowledge, only four resting-state fMRI studies have focused on PBD patients. Using independent component analysis (ICA) method, Wu et al. (2013) found that PBD-mania patients revealed altered affective, executive and sensorimotor resting state networks when comparing to normal controls [13]. In two previous studies, we also found that PBD-mania patients showed abnormal resting-state neuronal activities in the basal ganglia, parietal cortex, occipital cortex [14], and the ventral-affective and dorsal-cognitive circuits [15]. Finally, in the study of Dickstein et al. (2010), significantly decreased functional connectivity in the fronto-temporal circuit was revealed in euthymic PBD patients [16]. All these studies above have provided some evidence for the resting state brain functional changes in PBD, but none of them choosing PBD-depression as subjects. This could be a huge limitation since limited studies which carefully classify the episode states of adult $\mathrm{BD}$ have provided evidence to prove that different mood episodes may cause different functional abnormalities of brain $[17,18]$. Cerullo et al. (2012) found that the right amygdala was significantly more positively correlated with the left inferior frontal gyrus during mania and with the right insula during depression in $\mathrm{BD}$ patients [18]. In a task-related fMRI study, Liu et al. (2012) observed that mania was associated with decreased right rostral prefrontal cortex activation to fearful and neutral faces, while depression was associated with increased left orbitofrontal cortex activation to fearful faces in BD patients [17]. All these indicate that fMRI studies in PBD-depression patients are eagerly needed.

Resting state fMRI is a fabulous way to characterize the baseline brain activities [19] without any tasks which can minimize the effect of the external stimuli [20]. Prior evidence shows that task-related changes in neural activation may just represent less than $5 \%$ of the brain's total activity, while the majority of its resources have been used on task-independent, spontaneous neural activity, and resting state fMRI study is the best approach to evaluate it [16]. Regional homogeneity (ReHo) is a data-driven method which can be applied to process the resting state fMRI data through analyzing the temporal synchronization of the inter-regional fMRI signals of the whole brain [21]. ReHo is established on the base of that the blood oxygen level-dependent(BOLD) signal of a given voxel is temporally similar to its neighbors especially when the brain area is involved in a specific condition [22]. ReHo abnormalities (increase or decrease) may indicate the changes of temporal neural activities in the regional brain [23] and suggest an unbalanced local functionality or an uncompensatory reaction of the whole brain network [24]. This method has been widely used to elucidate the possible pathophysiological mechanisms underlying many child psychiatric disorders, such as PBD-mania [15], attention-deficit/hyperactivity disorder (ADHD) [25], and autism spectrum disorders [26].

Taken together, the aim of the present study was to explore baseline brain activity changes in PBD-depression patients by using ReHo.

\section{Methods}

\section{Participants}

Seventeen PBD-depression children and adolescents were recruited from the child and adolescent psychiatric clinic of the Second Xiangya Hospital of Central South University, Changsha, Hunan, P.R. China from July to December, 2012. Meanwhile, 18 age- and sex-matched healthy controls (HC) were also recruited through advertisements in public schools. The inclusion criteria for PBD-depression patients were as follows: 1) met the Diagnostic and Statistical Manual for Mental Disorders, Fourth Edition (DSM-IV) criteria for BD with current depression episode; 2) aged from 10 to 18 years old; 3) right-handedness; 4) Han nationality; 5) could follow the instructions to keep still during MRI scanning. The exclusion criteria for all participants included: 1) presence of major sensorimotor handicaps; 2) full-scale intelligence quotient (IQ) $<80 ; 3$ ) contraindications to perform the MRI scan, including the presence of metallic implants, retractors or braces, and claustrophobia; 4) with other mental disorders, such as schizophrenia, anorexia or bulimia nervosa, and learning disabilities; 5) alcohol or drug dependence or abuse; 6) active medical or neurological diseases; 7) history of electroconvulsive therapy (ECT). The present study was approved by the ethic committee of the Second Xiangya Hospital of Central South University. Written informed consent forms were obtained from all the participants and their guardians.

\section{Procedures}

\section{Demographic and clinical assessments}

The diagnoses were ascertained through the consensus by two board-certified child psychiatrists on the basis of clinical interviews and administrations of Schedule for Affective Disorders and Schizophrenia for School aged Children Present and Lifetime Versions (K-SADS-PL) [27]. The demographic and clinical data were collected using a self-designed questionnaire from all the participants. The 
Wechsler Abbreviated Scale of Intelligence (WASI) was used to assess the intellectual ability of all the patients and controls. The severity of mood symptoms was assessed using the Young Mania Rating Scale (YMRS) [28] and Mood and Feelings Questionnaire (MFQ) [29] on the day of MRI scanning. MFQ is a well- designed measure that is very widely used to assess the severity of depression symptoms in youth. It has 33 items and each item scores as $0=$ never, $1=$ sometimes, $2=$ often. The total score, in general, is associated with severity of depressive symptoms. The Chinese version of MFQ was introduced in our study, which was translated by Cao et al. (2009) [30]. The MFQ showed good psychometric properties and good internal consistency (Cronbach's alpha $=0.93$ ) in a Chinese sample of 2592 middle school students [30].

\section{MRI acquisition}

Magnetic resonance imaging scans were performed using a Siemens 3.0-T scanner (Allegra; Siemens Medical System) at the Magnetic Resonance Center belonging to Hunan Provincial People's Hospital. All the participants were asked to remain motionless and to keep eyes closed without thinking of anything in particular. A standard birdcage head coil was used, and the restraining foam pads were placed on two sides of the head to minimize head motion.

The regular axial three-dimensional T1-weighted images were acquired with a spoiled gradient recall sequence with the following imaging parameters: slice thickness $=1 \mathrm{~mm}$, Gap $=0 \mathrm{~mm}$, repetition time $(\mathrm{TR})=2300 \mathrm{~ms}$, echo time $(\mathrm{TE})=2.03 \mathrm{~ms}$, field of view $(\mathrm{FOV})=256 * 256 \mathrm{~mm} 2$, flip angle $=9^{\circ}$, matrix size $=256 * 256$, Slices $=176$.

An echo planar imaging sequence was used to obtain the functional images, the parameters were as follows: 30 axial slices, $\mathrm{TR}=2000 \mathrm{~ms}, \mathrm{TE}=30 \mathrm{~ms}$, matrix $=64 \times 64$, flip angle $=90^{\circ}, \mathrm{FOV}=240 * 240 \mathrm{~mm}^{2}$, slice thickness $=4 \mathrm{~mm}$, gap $=0.4 \mathrm{~mm}$. A total of 250 brain volumes were collected, resulting in a total scan time of $500 \mathrm{~s}$.

\section{Image processing}

The Statistical Parametric Mapping (SPM) 8 software package (http://www.fil.ion.ucl.ac.uk/spm) was used to preprocess the image data. The first ten images were deleted for the signal equilibration. Then the remaining images were conducted for slice acquisition correction and head motion correction. The fMRI data which had less than $1.0 \mathrm{~mm}$ of head motion and $1.0^{\circ}$ of angular rotation were included. Moreover, the mean framewise displacement (FD) was computed by averaging $\mathrm{FD}_{\mathrm{i}}$ from every time point for each subject [31]. There were no differences for the mean FD between groups $(t=0.413, p=0.682)$ (Table 1). Then the fMRI images were normalized to the standard Montreal Neurological Institute (MNI) template provided by SPM and resam- ple to the 3-mm isotropic voxels. A temporal filter $(0.01 \mathrm{~Hz}<\mathrm{f}<0.08 \mathrm{~Hz})$ were used to reduce the lowfrequency drift and physiological high frequency respiratory and cardiac noise $[21,32]$.

The acquisition of individual ReHo map was performed with REST software (http://www.resting-fmri.sourceforge. net). The Kendall's coefficient of concordance (KCC) was calculated to measure regional homogeneity of the time series of a given voxel with its nearest neighbor (26 voxels) in a voxel-wise way that had described by Zang et al. (2004) [22]. Then a mask (made from the MNI template to assure matching with the normalization step), in the REST software, was used to remove non-brain tissue and for standardization purposes. Each individual ReHo map was divided by its own global mean KCC value within the mask [33]. Finally, the data was smoothed with a Gaussian kernel of $4 \mathrm{~mm}$ full-width at half-maximum.

\section{Statistical analysis}

Between group differences in demographic variables were examined using independent two-sample $t$ tests for continuous variables and chi-square tests for categorical variables in Statistical Package for the Social Sciences (SPSS) version 16.0 (SPSS Inc., Chicago, IL, USA). The level of two-tailed statistical significance was set at $p<0.05$ for all tests.

To explore ReHo differences between PBD-depression patients and normal controls, a second-level random-effect two-sample $t$ test was performed in a voxel-by-voxel manner. Significant differences were set at a corrected significance level of $p<0.05$ [combined height threshold $p<0.01(\mathrm{~T}>2.46)$ and a minimum cluster size of 18 voxels]. Threshold correction was performed by using AlphaSim program (parameters were as follows: individual voxel $p=0.01,1000$ simulations, FWHM $=4 \mathrm{~mm}$, with mask) in the REST software, which applied Monte Carlo simulation to calculate the probability of false-positive detection by taking into consideration both the individual voxel probability thresholding and cluster size [34].

Furthermore, Pearson's correlation analyses were performed to explore the relationships between MFQ scores and mean ReHo values within regions displaying significant between-group differences in PBD-depression.

\section{Results}

\section{Demographic and clinical characteristics}

Demographic and clinical features of patients and healthy controls in the sample are presented in Table 1. There was no significant difference between two groups for age, gender, educational level, IQ and YMRS score. As expected, the mean MFQ score was significant higher in PBD-depression patients than controls. Nine(52.9\%) patients were free of medication at the time of MRI 
Table 1 Demographic and clinical characteristics of all subjects $(n=35)$

\begin{tabular}{|c|c|c|c|c|}
\hline & PBD-depression $n=17$ means(SD) & HC $n=18$ means (SD) & $t / x^{2}$ & $P$-values \\
\hline Age & $14.4(1.77)$ & $14.1(1.61)$ & 0.527 & 0.602 \\
\hline Gender (Male/Female) & $7 / 10$ & $6 / 12$ & 0.230 & 0.631 \\
\hline IQ & 102(14.6) & 105(7.72) & -0.808 & 0.425 \\
\hline YMRS score & $4.18(1.47)$ & $3.67(2.11)$ & 0.824 & 0.416 \\
\hline MFQ score & $30.3(9.67)$ & $6.39(3.36)$ & 9.878 & 0.000 \\
\hline Mean FD & $0.128(0.03)$ & $0.133(0.05)$ & 0.413 & 0.682 \\
\hline Illness duration (months) & $10.5(6.55)$ & & & \\
\hline Onset age (years) & 13.5(1.81) & & & \\
\hline Episode times & $3.88(2.53)$ & & & \\
\hline \multicolumn{5}{|l|}{ Medication, n (\%) } \\
\hline None & $9(52.9)$ & & & \\
\hline Lithium & $4(23.5)$ & & & \\
\hline Valproate & $4(23.5)$ & & & \\
\hline Antipsychotic drugs & $8(47.1)$ & & & \\
\hline Antidepressant & $2(11.8)$ & & & \\
\hline \multicolumn{5}{|l|}{ Comorbidity, n (\%) } \\
\hline Anxiety & $1(5.88)$ & & & \\
\hline $\mathrm{ADHD}$ & $2(11.8)$ & & & \\
\hline OCD & $1(5.88)$ & & & \\
\hline
\end{tabular}

ADHD, attention deficit hyperactivity disorder; FD, framewise displacement; IQ, intelligence quotient; MFQ, mood and feelings questionnaire; $\mathrm{HC}$, healthy controls; OCD, obsessive compulsive disorder; PBD, pediatric bipolar disorder; YMRS, Young Mania Rating Scale.

scanning; four(23.5\%) patients had comorbid disorder, particularly ADHD.

\section{Between-group ReHo differences}

As compared with the control group, PBD-depression patients showed significant decreased ReHo values in the medial frontal gyrus, bilateral middle frontal gyrus and middle temporal gyrus, and the right putamen (see Table 2 and Figure 1). However, there were no brain regions revealing increased $\mathrm{ReHo}$ values in patients.

Table 2 Brain regions showing decreased ReHo between PBD-depression patients and normal controls

\begin{tabular}{|c|c|c|c|c|c|c|}
\hline \multirow[t]{2}{*}{ Brain regions } & \multirow[t]{2}{*}{ Hemisphere } & \multirow[t]{2}{*}{$\begin{array}{l}\text { Cluster } \\
\text { size }\end{array}$} & \multicolumn{3}{|c|}{$\begin{array}{l}\text { MNI } \\
\text { coordinates }\end{array}$} & \multirow[t]{2}{*}{$T$ value } \\
\hline & & & $x$ & $y$ & $z$ & \\
\hline Medial frontal gyrus & & 74 & 0 & 6 & 48 & -4.98 \\
\hline \multirow[t]{2}{*}{ Middle frontal gyrus } & Left & 95 & -30 & -3 & 66 & -5.43 \\
\hline & Right & 70 & 42 & -12 & 39 & -5.30 \\
\hline \multirow[t]{2}{*}{ Middle temporal gyrus } & Left & 41 & -48 & -30 & -9 & -4.07 \\
\hline & Right & 34 & 57 & -21 & -9 & -3.90 \\
\hline Putamen & Right & 30 & 30 & -18 & 6 & -3.63 \\
\hline
\end{tabular}

MNI, Montreal Neurological Institute.cp.

\section{Correlations}

Within the PBD-depression patients, correlations of ReHo values in brain regions with significant between-group differences and MFQ scores were evaluated. Significant negative correlations were observed in the medial frontal gyrus $(r=-0.519, p=0.033)$ and the right middle frontal gyrus $(r=-0.627, p=0.007)$ (see Figure 2 ).

\section{Discussion}

In the present study, ReHo was used to show neural synchronization of local brain areas in PBD-depression patients during resting state. The current results suggest that diffuse brain regions with decreased ReHo values occur in PBD-depression. These regions are mainly distributed over the medial frontal gyrus, bilateral middle frontal gyrus and middle temporal gyrus, and the right putamen. Furthermore, in PBD-depression patients, there were significant negative correlations between MFQ scores and mean ReHo values in medial frontal gyrus and right middle frontal gyrus. Our findings showed that the abnormalities of baseline brain activities in PBD-depression are much more robust than those reported in adult $\mathrm{BD}$-depression [35], which may indicate that $\mathrm{BD}$ with early-onset could be a more severe clinic form. Consistent with our assumptions, the present findings are partly different from what we have found in our prior study for PBD-mania [15], suggesting that there 


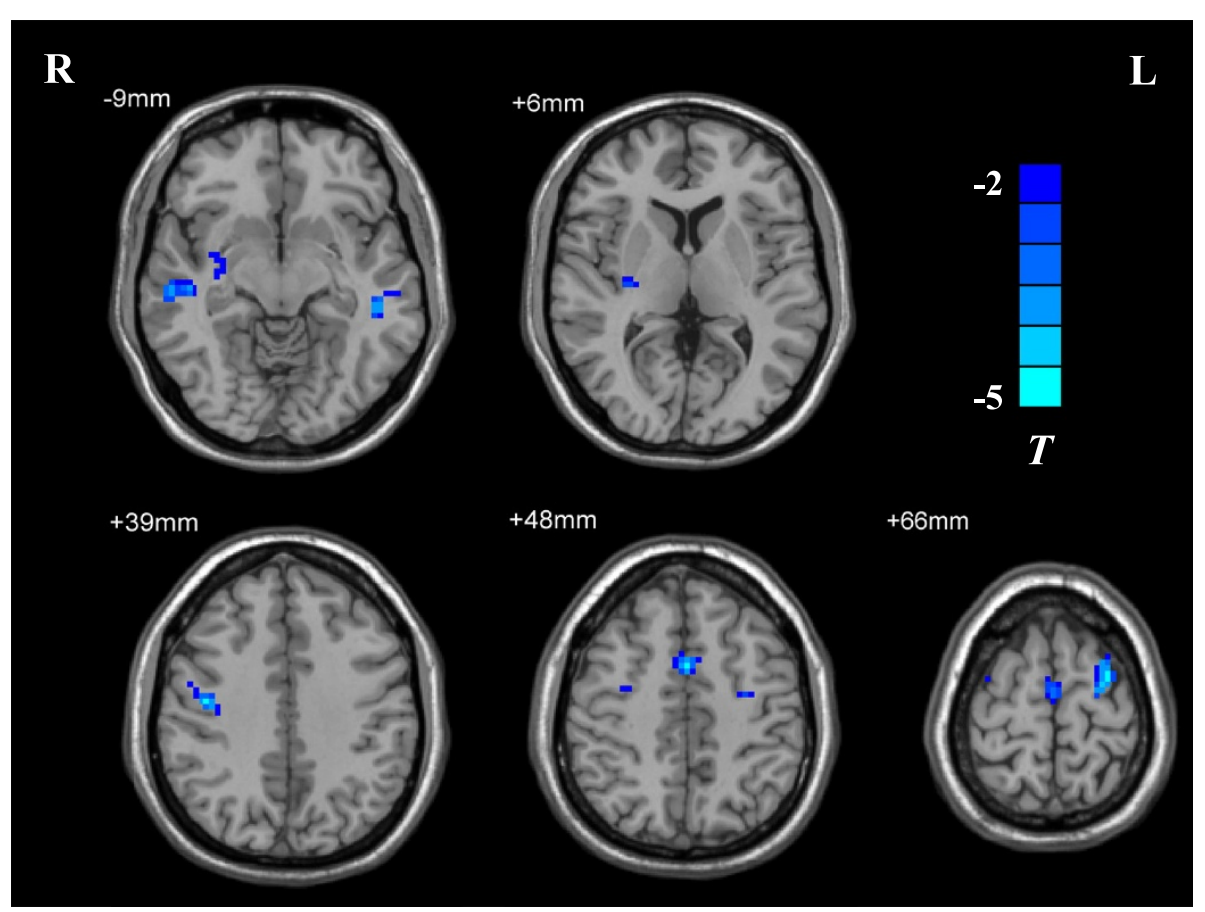

Figure 1 T-statistical different maps between PBD-depression patients and healthy controls (two-sample $t$ test; $p<0.05$, AlphaSim corrected). Regions with decreased ReHo values are shown in blue.

could be different brain functional alterations involving in different mood episodes.

In the present study, PBD-depression patients showed decreased ReHo values in frontal regions, including the medial frontal gyrus and the bilateral middle frontal gyrus. It has been reported that the medial prefrontal cortex (mPFC) plays an important role in the regulation and generation of emotion [36], which is based on the dense and reciprocal connectivity with subcortical regions, such as amygdala [37]. Previous studies have suggested the involvement of the medial frontal gyrus in the pathophysiology of BD. Our results are consistent with previous findings showing significantly decreased absolute regional metabolism in the medial frontal gyrus [38] and increased ReHo values in the left media frontal gyrus [35] in adult BD-depression patients. In addition, the present findings are also in agreement with a study that reported decreased connectivity in the MPFC in adult BD patients (13 with mania episode and 4 with mixed episode) [39].

A second noteworthy finding was the decreased ReHo values in bilateral middle frontal gyrus. The middle frontal gyrus has been reported to be involved in decision making, affective modulation, and conflict resolution $[40,41]$. In a prior study for patients with first-episode BD-mania, the middle frontal gyrus was abnormally activated following a response inhibition task, which suggested that the recruitment of the middle frontal gyrus might be considered as a compensatory neural strategy to manage the demands of this task [42]. In another positron emission tomography (PET) study for medication-free outpatients with BD-depression, decreased metabolism in bilateral middle frontal gyrus was negatively correlated with the severity of depression symptoms [38]. Moreover, structural MRI studies also demonstrated that gray matter volume was reduced in the right middle frontal gyrus in a group of patients with $\mathrm{BD}$ II [43]. Together with our results, these findings indicate that functional abnormality in the frontal gyrus is existed not only in adult BD but also in PBD. We propose that brain functional impairments of the frontal gyrus may exist in the early stage of BD and persist into adulthood.

Decreased ReHo was also found in bilateral middle temporal gyrus in patients with PBD-depression. The temporal lobe is a visual and auditory-related brain region that plays a vital role in working memory processing and facial emotion processing [44,45]. Structural and functional MRI studies have accumulated the evidence that $\mathrm{BD}$ is associated with the alterations in temporal lobe. Chen et al. (2007) [46] observed decreased gray matter volume in the left middle superior temporal gyrus in adult BD patients. In addition, volume reductions in the left superior and middle temporal gyrus in adult patients with BD II were reported by another study [47]. For functional studies, adult BD-depression showed significantly lowered regional cerebral blood flow in the anterior temporal 

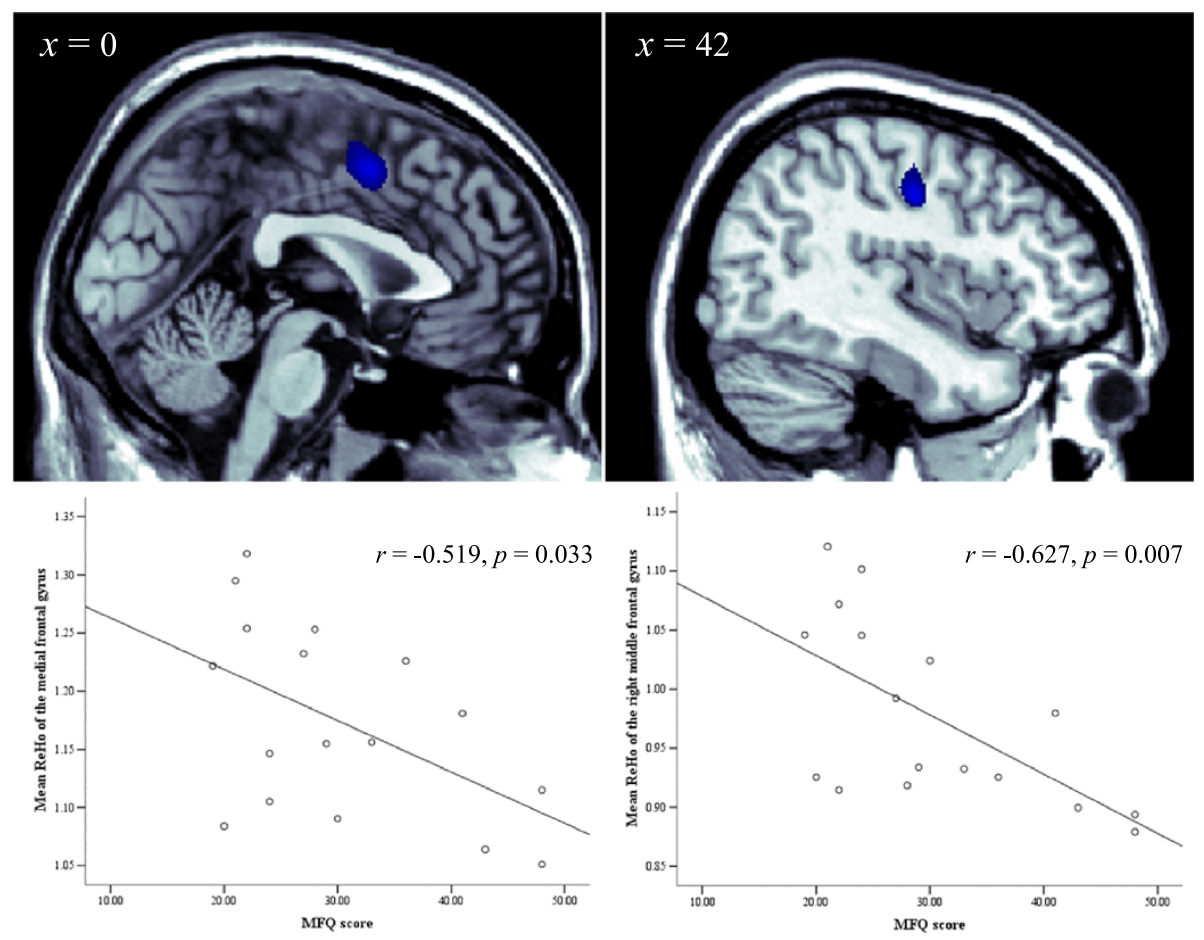

Figure 2 Correlation analysis between MFQ Scores and ReHo values in the PBD-depression patients $(p<0.05$, corrected).

regions bilaterally [48]. Decreased metabolism in the middle temporal gyrus was significantly associated with depression symptoms in a sample of medication-free patients with adult BD-depression [38]. Another restingstate study for adult BD-depression patients noted that the amplitude of low-frequency fluctuation (ALFF) was increased in bilateral temporal gyrus in the patient group [5]. Our result, in combination with these prior findings, suggests that the middle temporal gyrus is presumably part of a relevant functional network associated with BD, especially during a depressive episode.

The final region we found to show decreased ReHo was the right putamen. Putamen belongs to the lateral paralimbic system which is involved in the motivational processes and has connectivity to the mPFC $[36,49]$. Abnormal brain activity in the putamen has been reported in the task-related MRI studies in BD. Blumberg et al. (2003) [50] observed increased activation of the left putamen in ten adolescents with BD while they were performing an event-related fMRI color naming Stroop task. Consistent with this finding, Surguladze et al. (2010) [51] also found increased left putamen activity in response to moderate fear and high intensity happy faces in adult BD patients as compared with normal controls. Interestingly, an adult study including medication-free BD patients (stratify groups by depression, euthymia, and mania episode) performing a negative facial emotion matching task showed greater activations in the putamen in all three $\mathrm{BD}$ groups when comparing to controls [52]. Of note, even in symptom-free youths at a familial risk for $\mathrm{BD}$, this difference mentioned above has already occurred, which may indicate that brain functional changes in the putamen is a notably sensitive marker for early stage of BD [53]. Also functional abnormalities in the putamen may be considered as a clinic marker for early identification of PBD.

\section{Limitations}

Several limitations should be considered when interpreting the present results. First of all, the sample size in our study is relatively small, which may give rise to the problem that some subtle changes in the brain cannot be observed. Secondly, nearly half of the patients are taking medication when scanning, thus, medication exposure becomes a potential confound in this study. Thirdly, these results should be tested in a study which compares brain functional abnormalities of a same person with different mood states.

\section{Conclusions}

In conclusion, our results suggest that extensive regions with altered baseline brain activities are existed in PBDdepression and these brain regions mainly locate in the fronto-limbic circuit and associated striatal structures. Moreover, the present findings also add to our understanding that there could be unique neuropathophysiological mechanisms underlying PBD-depression. 


\section{Competing interests}

The authors declare that they have no competing interests.

\section{Authors' contributions}

Authors WG and LS designed this study and wrote the first draft of the manuscript. Authors $S L, Q X, D L$, and FY recruited the sample and finished the clinical assessment. Authors $Q J, Y Z$, and $R Q$ managed the data analysis. Authors GL and LS also designed the study and had full access to all of the data in the study and take responsibility for the integrity of the data and the accuracy of the data analysis. All authors contributed to and have approved the final manuscript.

\section{Acknowledgments}

This work was supported by the National Nature Science Foundation of China (81371531, 81171291 and 81201077), Key Program for Guangming Lu (BWS11J063, and 10z026), and Humanity and Social Science Youth foundation of Ministry of Education (11YJC190039). Besides, the authors would like to thank all participants who took part in this study, and the experts at the Magnetic Resonance Center of Hunan Provincial People's Hospital for providing scan time and technical assistant.

\section{Author details}

${ }^{1}$ Mental Health Institute of The Second Xiangya Hospital, National Technology Institute of Psychiatry, Key Laboratory of Psychiatry and Mental Health of Hunan Province, Central South University, No. 139 Renmin Road, Changsha 410011, Hunan, China. ${ }^{2}$ Department of Child Psychology, The Children's Hospital, Zhejiang University School of Medicine, Hangzhou, Zhejiang, China. ${ }^{3}$ Department of Radiology, Taishan Medical University, Taian, Shandong, China. ${ }^{4}$ Department of Mental Health, First Affiliated Hospital, Zhejiang University School of Medicine, Hangzhou, Zhejiang, China. ${ }^{5}$ Department of Medical Imaging, Jinling Hospital, Clinical School of Medical College, Nanjing University, 305 Zhongshan East Road, Nanjing 210002, Jiangsu, China. ${ }^{6}$ Department of Psychology, Nanjing Normal University, Nanjing, Jiangsu, China.

Received: 20 March 2014 Accepted: 23 July 2014 Published: 6 August 2014

\section{References}

1. Schneider MR, DelBello MP, McNamara RK, Strakowski SM, Adler CM: Neuroprogression in bipolar disorder. Bipolar Disord 2012, 14(4):356-374.

2. Perlis RH, Miyahara S, Marangell LB, Wisniewski SR, Ostacher M, DelBello MP, Bowden CL, Sachs GS, Nierenberg AA: Long-term implications of early onset in bipolar disorder: data from the first 1000 participants in the systematic treatment enhancement program for bipolar disorder (STEP-BD). Biol Psychiatry 2004, 55(9):875-881.

3. Mitchell PB, Goodwin GM, Johnson GF, Hirschfeld RM: Diagnostic guidelines for bipolar depression: a probabilistic approach. Bipolar Disord 2008, 10(1 Pt 2):144-152.

4. Judd LL, Akiskal HS: Depressive episodes and symptoms dominate the longitudinal course of bipolar disorder. Curr Psychiatry Rep 2003, 5(6):417-418.

5. Liu CH, Li F, Li SF, Wang YJ, Tie CL, Wu HY, Zhou Z, Zhang D, Dong J, Yang Z, Wang $C Y$ : Abnormal baseline brain activity in bipolar depression: a resting state functional magnetic resonance imaging study. Psychiatry Res 2012, 203(2-3):175-179.

6. Strakowski SM, Delbello MP, Adler CM: The functional neuroanatomy of bipolar disorder: a review of neuroimaging findings. Mol Psychiatry 2005, 10(1):105-116.

7. Strakowski SM, Adler CM, Almeida J, Altshuler LL, Blumberg HP, Chang KD, DelBello MP, Frangou S, McIntosh A, Phillips ML, Sussman JE, Townsend JD: The functional neuroanatomy of bipolar disorder: a consensus model. Bipolar Disord 2012, 14(4):313-325.

8. Foland-Ross LC, Bookheimer SY, Lieberman MD, Sugar CA, Townsend JD, Fischer J, Torrisi S, Penfold C, Madsen SK, Thompson PM, Altshuler LL: Normal amygdala activation but deficient ventrolateral prefrontal activation in adults with bipolar disorder during euthymia. Neuroimage 2012, 59(1):738-744.

9. Wessa M, Kanske P, Linke J: Bipolar disorder: A neural network perspective on a disorder of emotion and motivation. Restor Neurol Neurosci 2014, 32(1):51-62.
10. Passarotti AM, Sweeney JA, Pavuluri MN: Fronto-limbic dysfunction in mania pre-treatment and persistent amygdala over-activity post-treatment in pediatric bipolar disorder. Psychopharmacology (Berl) 2011, 216(4):485-499.

11. Anand A, Li Y, Wang Y, Lowe MJ, Dzemidzic M: Resting state corticolimbic connectivity abnormalities in unmedicated bipolar disorder and unipolar depression. Psychiatry Res 2009, 171(3):189-198.

12. Versace A, Thompson WK, Zhou D, Almeida JR, Hassel S, Klein CR, Kupfer DJ, Phillips ML: Abnormal left and right amygdala-orbitofrontal cortical functional connectivity to emotional faces: state versus trait vulnerability markers of depression in bipolar disorder. Biol Psychiatry 2010, 67(5):422-431.

13. Wu M, Lu LH, Passarotti AM, Wegbreit E, Fitzgerald J, Pavuluri MN: Altered affective, executive and sensorimotor resting state networks in patients with pediatric mania. J Psychiatry Neurosci 2013, 38(4):232-240.

14. Lu D, Jiao Q, Zhong Y, Gao W, Xiao Q, Liu X, Lin X, Cheng W, Luo L, Xu C, Lu G, Su L: Altered baseline brain activity in children with bipolar disorder during mania state: a resting-state study. Neuropsychiatr Dis Treat 2014, 10:317-323.

15. Xiao Q, Zhong Y, Lu D, Gao W, Jiao Q, Lu G, Su L: Altered regional homogeneity in pediatric bipolar disorder during manic state: a resting-state fMRI study. PLoS One 2013, 8(3):e57978.

16. Dickstein DP, Gorrostieta C, Ombao H, Goldberg LD, Brazel AC, Gable CJ, Kelly C, Gee DG, Zuo XN, Castellanos FX, Milham MP: Fronto-temporal spontaneous resting state functional connectivity in pediatric bipolar disorder. Biol Psychiatry 2010, 68(9):839-846.

17. Liu J, Blond BN, van Dyck LI, Spencer L, Wang F, Blumberg HP: Trait and state corticostriatal dysfunction in bipolar disorder during emotional face processing. Bipolar Disord 2012, 14(4):432-441.

18. Cerullo MA, Fleck DE, Eliassen JC, Smith MS, DelBello MP, Adler CM, Strakowski SM: A longitudinal functional connectivity analysis of the amygdala in bipolar I disorder across mood states. Bipolar Disord 2012, 14(2):175-184

19. Qiu YW, Han L, Lv XF, Jiang GH, Tian JZ, Zhuo FZ, Su HH, Lin CL, Zhang XL: Regional homogeneity changes in heroin-dependent individuals: resting-state functional MR imaging study. Radiology 2011, 261(2):551-559.

20. Liao H, Wang L, Zhou B, Tang J, Tan L, Zhu X, Yi J, Chen X, Tan C: $A$ resting-state functional magnetic resonance imaging study on the first-degree relatives of persons with schizophrenia. Brain Imaging Behav 2012, 6(3):397-403.

21. Chen HJ, Zhu XQ, Yang M, Liu B, Zhang Y, Wang Y, Teng GJ: Changes in the regional homogeneity of resting-state brain activity in minimal hepatic encephalopathy. Neurosci Lett 2012, 507(1):5-9.

22. Zang $Y$, Jiang $T, L u Y, H e Y$, Tian L: Regional homogeneity approach to fMRI data analysis. Neuroimage 2004, 22(1):394-400.

23. Zhong Y, Lu G, Zhang Z, Jiao Q, Li K, Liu Y: Altered regional synchronization in epileptic patients with generalized tonic-clonic seizures. Epilepsy Res 2011, 97(1-2):83-91.

24. Dai XJ, Gong HH, Wang YX, Zhou FQ, Min YJ, Zhao F, Wang SY, Liu BX, Xiao $X Z$ : Gender differences in brain regional homogeneity of healthy subjects after normal sleep and after sleep deprivation: a resting-state fMRI study. Sleep Med 2012, 13(6):720-727.

25. Zhu CZ, Zang YF, Cao QJ, Yan CG, He Y, Jiang TZ, Sui MQ, Wang YF: Fisher discriminative analysis of resting-state brain function for attention-deficit/ hyperactivity disorder. Neuroimage 2008, 40(1):110-120.

26. Paakki JJ, Rahko J, Long X, Moilanen I, Tervonen O, Nikkinen J, Starck T, Remes J, Hurtig T, Haapsamo H, Jussila K, Kuusikko-Gauffin S, Mattila ML, Zang Y, Kiviniemi V: Alterations in regional homogeneity of resting-state brain activity in autism spectrum disorders. Brain Res 2010, 1321:169-179.

27. Kaufman J, Birmaher B, Brent D, Rao U, Flynn C, Moreci P, Williamson D, Ryan N: Schedule for Affective Disorders and Schizophrenia for School-Age Children-Present and Lifetime Version (K-SADS-PL): initial reliability and validity data. J Am Acad Child Adolesc Psychiatry 1997, 36(7):980-988.

28. Young RC, Biggs JT, Ziegler VE, Meyer DA: A rating scale for mania: reliability, validity and sensitivity. Br J Psychiatry 1978, 133:429-435.

29. Wood A, Kroll L, Moore A, Harrington R: Properties of the mood and feelings questionnaire in adolescent psychiatric outpatients: a research note. J Child Psychol Psychiatry 1995, 36(2):327-334.

30. Cao F, Su L, Cheng P: Reliability and validity of the Mood and Feelings Questionnaire in Chinese adolescents. Chinese Journal of Clinical Psychology 2009, 17(4):440-442. 
31. Liao W, Zhang Z, Mantini D, Xu Q, Wang Z, Chen G, Jiao Q, Zang YF, Lu G: Relationship between large-scale functional and structural covariance networks in idiopathic generalized epilepsy. Brain connectivity 2013, 3(3):240-254.

32. He $Y$, Wang $L$, Zang $Y$, Tian $L$, Zhang $X$, Li K, Jiang T: Regional coherence changes in the early stages of Alzheimer's disease: a combined structural and resting-state functional MRI study. Neuroimage 2007, 35(2):488-500.

33. Wu T, Long X, Zang Y, Wang L, Hallett M, Li K, Chan P: Regional homogeneity changes in patients with Parkinson's disease. Hum Brain Mapp 2009, 30(5):1502-1510.

34. Poline JB, Worsley KJ, Evans AC, Friston KJ: Combining spatial extent and peak intensity to test for activations in functional imaging. Neuroimage 1997, 5(2):83-96.

35. Liu CH, Ma X, Li F, Wang YJ, Tie CL, Li SF, Chen TL, Fan TT, Zhang Y, Dong J, Yao L, Wu X, Wang CY: Regional homogeneity within the default mode network in bipolar depression: a resting-state functional magnetic resonance imaging study. PLOS One 2012, 7(11):e48181.

36. Kober H, Barrett LF, Joseph J, Bliss-Moreau E, Lindquist K, Wager TD: Functional grouping and cortical-subcortical interactions in emotion: a meta-analysis of neuroimaging studies. Neuroimage 2008, 42(2):998-1031.

37. Anticevic A, Brumbaugh MS, Winkler AM, Lombardo LE, Barrett J, Corlett PR, Kober H, Gruber J, Repovs G, Cole MW, Krystal JH, Pearlson GD, Glahn DC: Global prefrontal and fronto-amygdala dysconnectivity in bipolar I disorder with psychosis history. Biol Psychiatry 2013, 73(6):565-573.

38. Brooks JO 3rd, Wang PW, Bonner JC, Rosen AC, Hoblyn JC, Hill SJ, Ketter TA: Decreased prefrontal, anterior cingulate, insula, and ventral striatal metabolism in medication-free depressed outpatients with bipolar disorder. J Psychiatr Res 2009, 43(3):181-188.

39. Ongur D, Lundy M, Greenhouse I, Shinn AK, Menon V, Cohen BM, Renshaw PF: Default mode network abnormalities in bipolar disorder and schizophrenia. Psychiatry Res 2010, 183(1):59-68.

40. Rogers RD, Owen AM, Middleton HC, Williams EJ, Pickard JD, Sahakian BJ, Robbins TW: Choosing between small, likely rewards and large, unlikely rewards activates inferior and orbital prefrontal cortex. J Neurosci 1999, 19(20):9029-9038.

41. Pochon JB, Levy R, Fossati P, Lehericy S, Poline JB, Pillon B, Le Bihan D, Dubois $B$ : The neural system that bridges reward and cognition in humans: an fMRI study. Proc Natl Acad Sci U S A 2002, 99(8):5669-5674.

42. Strakowski SM, Adler CM, Cerullo M, Eliassen JC, Lamy M, Fleck DE, Lee JH, DelBello MP: Magnetic resonance imaging brain activation in first-episode bipolar mania during a response inhibition task. Early Interv Psychiatry 2008, 2(4):225-233.

43. Ambrosi E, Rossi-Espagnet MC, Kotzalidis GD, Comparelli A, Del Casale A, Carducci F, Romano A, Manfredi G, Tatarelli R, Bozzao A, Girardi P: Structural brain alterations in bipolar disorder II: A combined voxel-based morphometry (VBM) and diffusion tensor imaging (DTI) study. J Affect Disord 2013, 150(2):610-615.

44. Antonius D, Prudent V, Rebani Y, D'Angelo D, Ardekani BA, Malaspina D, Hoptman MJ: White matter integrity and lack of insight in schizophrenia and schizoaffective disorder. Schizophr Res 2011, 128(1-3):76-82.

45. Pavuluri MN, Passarotti AM, Fitzgerald JM, Wegbreit E, Sweeney JA: Risperidone and divalproex differentially engage the fronto-striato-temporal circuitry in pediatric mania: a pharmacological functional magnetic resonance imaging study. J Am Acad Child Adolesc Psychiatry 2012, 51(2):157-170. e5.

46. Chen X, Wen W, Malhi GS, Ivanovski B, Sachdev PS: Regional gray matter changes in bipolar disorder: a voxel-based morphometric study. Aust N Z J Psychiatry 2007, 41(4):327-336.

47. Bruno SD, Papadopoulou K, Cercignani M, Cipolotti L, Ron MA: Structural brain correlates of IQ changes in bipolar disorder. Psychol Med 2006, 36(5):609-618

48. Bhardwaj $R$, Chakrabarti S, Mittal BR, Sharan P: A single photon emission computerized tomography (SPECT) study of regional cerebral blood flow in bipolar disorder. World J Biol Psychiatry 2010, 11 (2 Pt 2):334-343.

49. Ongur D, Price JL: The organization of networks within the orbital and medial prefrontal cortex of rats, monkeys and humans. Cereb Cortex 2000, 10(3):206-219.

50. Blumberg HP, Martin A, Kaufman J, Leung HC, Skudlarski P, Lacadie C, Fulbright RK, Gore JC, Charney DS, Krystal JH, Peterson BS: Frontostriatal abnormalities in adolescents with bipolar disorder: preliminary observations from functional MRI. Am J Psychiatry 2003, 160(7):1345-1347.
51. Surguladze SA, Marshall N, Schulze K, Hall MH, Walshe M, Bramon E, Phillips ML, Murray RM, McDonald C: Exaggerated neural response to emotional faces in patients with bipolar disorder and their first-degree relatives. Neuroimage 2010, 53(1):58-64.

52. Hulvershorn LA, Karne H, Gunn AD, Hartwick SL, Wang Y, Hummer TA, Anand A: Neural activation during facial emotion processing in unmedicated bipolar depression, euthymia, and mania. Biol Psychiatry 2012, 71(7):603-610.

53. Deveney CM, Connolly ME, Jenkins SE, Kim P, Fromm SJ, Brotman MA, Pine DS, Leibenluft E: Striatal dysfunction during failed motor inhibition in children at risk for bipolar disorder. Prog Neuropsychopharmacol Biol Psychiatry 2012, 38(2):127-133.

\section{doi:10.1186/s12888-014-0222-y}

Cite this article as: Gao et al.: Alterations of regional homogeneity in pediatric bipolar depression: a resting-state fMRI study. BMC Psychiatry 2014 14:222.

\section{Submit your next manuscript to BioMed Central and take full advantage of:}

- Convenient online submission

- Thorough peer review

- No space constraints or color figure charges

- Immediate publication on acceptance

- Inclusion in PubMed, CAS, Scopus and Google Scholar

- Research which is freely available for redistribution 